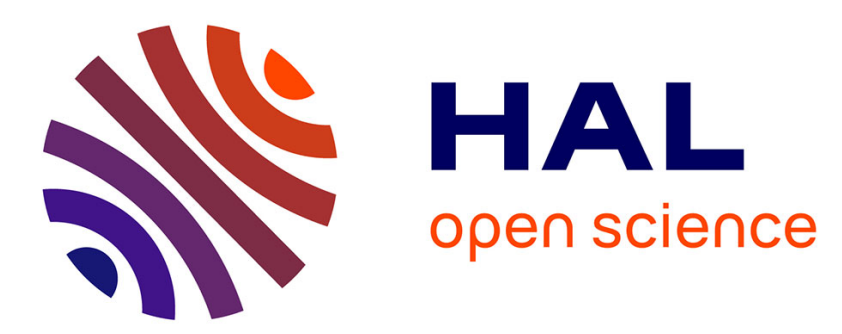

\title{
Quelles légitimités pour les services fonctionnels de la communication et de la formation dans les collectivités locales?
}

Dominique Bessières, François Grima

\section{- To cite this version:}

Dominique Bessières, François Grima. Quelles légitimités pour les services fonctionnels de la communication et de la formation dans les collectivités locales?. Politiques et Management public, 1999, 17, pp. 23-38. halshs-00838953

\section{HAL Id: halshs-00838953 \\ https://shs.hal.science/halshs-00838953}

Submitted on 26 Jun 2013

HAL is a multi-disciplinary open access archive for the deposit and dissemination of scientific research documents, whether they are published or not. The documents may come from teaching and research institutions in France or abroad, or from public or private research centers.
L'archive ouverte pluridisciplinaire HAL, est destinée au dépôt et à la diffusion de documents scientifiques de niveau recherche, publiés ou non, émanant des établissements d'enseignement et de recherche français ou étrangers, des laboratoires publics ou privés. 


\section{Quelles légitimités pour les services fonctionnels de la}

\section{communication et de la formation dans les collectivités locales ?}

In: Politiques et management public, vol. $17 \mathrm{n}^{\circ} 4$, 1999. pp. 23-38.

\section{Résumé}

Les collectivités locales sont des organisations complexes : des compétences diverses, soumises au principe hiérarchique, avec une dualité administration et élus. Les actions transversales des services fonctionnels de la communication et de la formation sont des moyens d'instaurer une cohérence. Mais, la question de la légitimité de leur existence est posée dans un contexte économique tendu parce qu'ils sont récents et que l'évaluation de leurs résultats est difficile. Aussi, l'enjeu de l'analyse réside dans la recherche des conditions de pérennisation de ces activités : quels sont les registres de la légitimation professionnelle ? Elles ont trait à la manifestation du pouvoir de l'élu, à une logique de service visant à constituer une expertise, à la mobilisation d'un environnement extérieur légitimateur pourvoyeur de reconnaissance légale ou professionnelle. L'affirmation de tels services recouvre des enjeux de pouvoir politique et de management public.

Citer ce document / Cite this document :

Bessières Dominique, Grima François. Quelles légitimités pour les services fonctionnels de la communication et de la formation dans les collectivités locales ?. In: Politiques et management public, vol. 17 n 4, 1999. pp. 23-38.

doi : $10.3406 /$ pomap.1999.2250

http://www.persee.fr/web/revues/home/prescript/article/pomap_0758-1726_1999_num_17_4_2250 


\title{
QUELLES LÉGITIMITÉS POUR LES SERVICES FONCTIONNELS DE LA COMMUNICATION ET DE LA FORMATION DANS LES COLLECTIVITÉS LOCALES?
}

\author{
Dominique BESSIĖRES * \\ François GRIMA **
}

Résumé

\begin{abstract}
Les collectivités locales sont des organisations complexes: des compétences diverses, soumises au principe hiérarchique, avec une dualité administration et élus. Les actions transversales des services fonctionnels de la communication et de la formation sont des moyens d'instaurer une cohérence. Mais, la question de la légitimité de leur existence est posée dans un contexte économique tendu parce qu'ils sont récents et que l'évaluation de leurs résultats est difficile. Aussi, l'enjeu de l'analyse réside dans la recherche des conditions de pérennisation de ces activités: quels sont les registres de la légitimation professionnelle ? Elles ont trait à la manifestation du pouvoir de l'élu, à une logique de service visant à constituer une expertise, à la mobilisation d'un environnement extérieur légitimateur pourvoyeur de reconnaissance légale ou professionnelle. L'affirmation de tels services recouvre des enjeux de pouvoir politique et de management public.
\end{abstract}

- Université Paris I Panthéon-Sorbonne.

* Université Evry-Val d'Essonne.

Revue POLITIQUES ET MANAGEMENT PUBLIC, Volume 17, n 4, décembre 1999.

(C) Institut de Management Public - 1999. 
Comme le soulignaient déjà Lawrence et Lorsch en 19671, les organisations doivent articuler un nombre croissant de fonctions différentes. Cette spécialisation des tâches, déjà mise à jour par Emile Durkheim à la fin du dix-neuvième siècle², engendre des tensions centripètes au sein des structures. Ce processus conduit à un morcellement des organisations entre différentes activités, instituant autant de "frontières infraorganisationnelles". La cohérence globale de l'organisation en est affectée, des difficultés de gestion en résultent.

A ce niveau réside lintérêt des services fonctionnels. Bien que l'expression demeure floue dans la littérature des sciences de gestion, deux dimensions peuvent être dégagées. Premièrement, ils se caractérisent structurellement par leur proximité du pouvoir central et leur positionnement en dehors de la ligne hiérarchique opérationnelle. Deuxièmement, ils ont pour rôle de constituer une expertise au sein de l'organisation dans un champ de compétences spécialisées. Ils veillent à I'homogénéité des pratiques par les services qu'ils proposent ${ }^{3}$. Ces vecteurs de cohérence sont fondamentalement horizontaux, transversaux. Cette dialectique entre un axe hiérarchique et une démarche de prestataire interne place ces services dans une position frontalière. Loin d'être une périphérie ne bénéficiant que du soutien du centre 4 de l'organisation pour s'imposer, le service frontalier dispose d'une dynamique propre issue de ce positionnement d'entre-deux 5 . II s'agit pour lui de faire cohabiter des logiques d'actions différentes et pouvant être contradictoires'.

Si ces postes fonctionnels peuvent apparaitre pertinents pour l'efficience de l'organisation, plusieurs facteurs viennent remettre en cause la légitimité de leur existence. D'un point de vue économique comme organisationnel, l'évaluation de leur valeur ajoutée propre se trouve entravée par la nécessaire imbrication avec les services opérationnels lors de la réalisation de leur action. En outre, pour pérenniser leur existence, ils se doivent de faire oublier le pouvoir discrétionnaire marquant leur origine. Cette configuration se retrouve pleinement dans le cas des fonctions de communication et de formation au sein des collectivités locales?.

1 Laurence (Paul), Lorsch (Jay), Organization and environment, Cambridge Mass, 1967.

2 Durkheim (Emile), De la division du travail social, (passim). Paris, PUF, coll. Bibliotheque philosophique contemporaine, 1967, 416 p. (notamment l'introduction pp. 1-11).

3 Malleret-Dumontant (Véronique). Une approche de la performance des services fonctionnels - L'évolution des centres de coûts discrétionnaires. Thèse pour le doctorat de Sciences de Gestion, Université Paris-IXDauphine, 1993.

4 Grémion (Pierre), Le pouvoir périphérique, Paris, Seuil, 1976, p. 11.

5 Pineau (Gaston), Les combats aux frontières des organisations, Montréal, Editions Sciences et Culture, $1980, . p .53$.

6 Katz et Khan, The social psychology of organisation, John Wiley and sons, New-York, 1978, 817 p.

7 Bessières (Dominique), L'institutionnalisation de la Communication Locale - Les cas des échelons décentralisés départementaux, régionaux, parisiens, franciliens. Thèse pour le doctorat de Science Politique, Université Paris-l-Panthéon-Sorbonne, juin 1998, 540 p. ; Grima (François), "Caractérisation et conséquences de l'activité frontalière en termes d'implication et de tension de rôle : le cas du responsable de formation", Congrès AGRH, novembre 1998. 
Sous l'effet d'un contexte organisationnel pluridimensionnel et de la spécificité de ces fonctions, la situation est encore plus complexe. Trois cacactéristiques définissent les administrations décentralisalisées : diversité des activités, principe hiérarchique, dualité administration-élus. Premièrement, les administrations décentralisées se caractérisent par une extrême variété des missions gérées (voirie, action sociale, éducation, culture...). Elles n'ont pas de cohérence intrinsèque. Deuxièmement, contrairement aux entreprises privées, elles sont régies strictement par un principe hiérarchique conditionnant les différents niveaux de la fonction publique territoriale sous l'égide d'un pouvoir politique fortement représentatif affirmé par la science politique ${ }^{1}$, aboutissant à un cloisonnement peu favorable aux fonctions transversales. Troisièmement, la dualité "structures administratives-élus" placés à leur tête débouche sur des tensions entre une logique gestionnaire et l'expression d'un pouvoir politique.

La communication comme la formation ne bénéficient pas d'une légitimité historique issue de l'antériorité, à l'image des directions financières. Elles ont une faible reconnaissance interne. D'autre part, elles traduisent la prise en compte par l'organisation d'enjeux sociétaux² dont l'intégration est décidée par les élus. En conséquence, le souci de leur évaluation et le travail d'oubli du poids du pouvoir central dans leur développement initial sont plus aigus, d'autant plus que la contrainte financière conduit à contrôler les charges de structure.

Dès lors, on peut s'interroger sur les différents registres de légitimation à l'œuvre dans ces fonctions afin d'assurer leur pérennité, voire d'étendre leur sphère d'influence dans l'organisation. Contrairement à la perspective de la sociologie fonctionnaliste ${ }^{3}$ qui souligne la difficulté d'exercice d'un rôle frontalier, nous mettrons en lumière qu'il existe des possibilités de tenir un tel positionnement sans ressentir de tensions inhibantes. Cependant, il ne s'agit pas pour autant de passer d'un extrême à l'autre. Nous ne prétendons pas que le positionnement frontalier procure à l'acteur une source de pouvoir incontestable4. Sa situation demeure fragile 5 . Les besoins de l'organisation peuvent évoluer et remettre en cause la pertinence de son apport dans le fonctionnement de celle-ci. Pour y demeurer, il doit faire la preuve à ses différents partenaires qu'il est intéressant de recourir à ses services.

Le but de cette contribution est d'analyser les conditions d'institutionnalisation de telles fonctions transversales récentes. Nous mobiliserons un cadre théorique intégrant les

1 Biarez (Sylvie), Le pouvoir local, Paris, Economica, 1989, 309 p. ; Meny (Yves), "La République des fiefs", in L'état de la décentralisation. Paris, La Documentation Française, Cahiers français n' 256, mai-juin 1992, pp. 60-65 ; Thoenig (Jean-Claude), "La décentralisation dix ans après", in L'état de la décentralisation. Paris, La Documentation Française, Cahiers français $n^{\circ} 256$, mai-juin 1992, pp. 73-80.

2 Thèmes de la société de communication et de la deuxième chance par la formation professionnelle continue.

3 Merton (R. K.), Eléments de théorie et de méthode sociologique, Paris, Plon, 1949.

4 Crozier (Michel), Le phénomène bureaucratique, Paris. Seuil, 1963, 381 p. ; Gould (R.). Fernandy (R.). "Structuration of mediation : formal approach to brokerage in transaction networks", in Sociological methodology, New-York, 1989 ; Degenne (A.), Frorsé (M.). Les réseaux sociaux, Paris, Armand Colin, 1994 ; Marsden ( $P$. $V$.). "Brokerage behaviors in restricted exchange networks", in Social structure and network analysis, New-York, Sage, 1982.

5 Pineau (Gaston), op. cit. 
apports de la science politique et des sciences de l'organisation. En effet, afin de saisir la complexité de cette dynamique d'adaptation, nous combinerons une approche structurelle d'inspiration politiste et une grille de lecture fondée sur l'individu dans le champ des sciences de l'organisation. Nous conjuguerons ainsi les analyses institutionnelles et managériales. Notre étude empirique s'articule autour de deux séries d'entretiens semi-directifs auprès respectivement de responsables de la communication d'une part, et de la formation professionnelle continue d'autre part, au sein des trois niveaux de collectivités territoriales (mairies, départements, région) franciliens. De plus, spécifiquement pour le second terrain afin d'atteindre une dimension nationale, une analyse quantitative complémentaire fut réalisée. Cette démarche heuristique permet une appréhension fine des processus d'implantation de nouvelles fonctions transversales au sein des collectivités locales.

\section{Une intégration délicate en construction}

L'existence des services fonctionnels dans les organisations est sensée améliorer leur efficience globale et faciliter la constitution, l'entretien, d'une expertise dans un champ de compétences précis. Luttant contre la compartimentation intrinsèque des collectivités locales éclatées entre de multiples activités ${ }^{1}$ distinctes, les services fonctionnels veillent à maintenir une certaine cohésion entre leurs composantes opérationnelles. Paradoxalement, si ce travail englobant d'unification justifie leur intégration, il rend leur acceptation difficile. Ils sont perçus comme des activités non directement "productives" à la rationalité économique incertaine, en raison du problème de leur évaluation. L'ampleur de leur champ d'intervention, la difficulté de l'évaluation de leurs apports spécifiques contribuent à poser le problème de leur utilité dans le fonctionnement des organisations. Ces difficultés communes aux services fonctionnels se trouvent aggravées par le caractère récent de leur intégration.

\section{- Des fonctions récentes au prise avec un contexte économique tendu}

La communication comme la formation, en tant que services administratifs, ont intégré récemment les collectivités locales. Leur institutionnalisation est loin d'être aboutie?.

Concernant, la communication, son antériorité historique remonte pour ses exemples les plus anciens aux années 1960. Elle se manifeste avec l'apparition des premiers bulletins municipaux de faible diffusion et périodicité. Les années soixante-dix sont celles de la constitution des premiers services de communication au sein des grandes

$1 \mathrm{~V}$. pour la description des registres de compétences et leurs évolutions Blanc (Jacques), Remond (Bruno), Les collectivités locales, Paris, Presses de la Fondation Nationale des Sciences Politiques et Dalloz, coll. Amphithéâtre, 1995, (3è édition), 689 p.

2 Bessières (Dominique), op. cit ; Layole (Gérard), "Formation : la fin du toujours plus ou comment faire mieux", Etude Entreprise et Personnel, septembre 1994. 
villes1, mais de façon non exhaustive. Après les élections municipales de 1977 et surtout dans les années quatre-vingt, généralement, on rencontre des services de communication placés sous l'autorité du maire ou du directeur de cabinet et dirigés par des professionnels². II est incontestable que la décentralisation a accru le phénomène.

De façon similaire, les services de formation ont connu quelques exemples dès les années soixante, mais le mouvement de création des services n'est significatif au sein des administrations décentralisés qu'à partir des années quatre-vingt et surtout au début des années quatre-vingt-dix ${ }^{3}$.

La jeunesse de ces fonctions se retrouve dans le statut professionnel des personnes les mettant en œuvre dans les organisations. Dans les deux cas, il est clairement marqué par la fragilité des positions acquises. Les responsables de la communication sont majoritairement des contractuels dont l'insertion professionnelle dans la durée dépend de la volonté du pouvoir exécutif. Ce positionnement dans une organisation fonctionnarisée les prive de possibilités d'évolution de carrière au sein de la même institution. De plus, la faiblesse de la définition des modalités d'exercice du métier, la jeunesse des formations spécialisées en ce domaine, concourent à renforcer le pouvoir de l'élu dans la relation salariale. En effet, des concours spécifiques à cette fonction semblent pour l'heure exclus : les compétences requises pour ces postes sont rares 4 . Les responsables de formation ont une position moins précaire. Les postes sont tenus majoritairement par des fonctionnaires titulaires (attachés territoriaux $(42,8 \%)$, rédacteurs $(33 \%)$, contractuels $\left.(7,2 \%)^{5}\right)$. Ils exercent ici leur première responsabilité professionnelle. Cette faible ancienneté dans la fonction, synonyme d'expertise limitée dans le champ de l'ingéniérie de formation, limite leur crédibilité professionnelle. Ne pouvant s'appuyer sur la reconnaissance acquise de leur fonction et ne disposant pas de capital technique suffisamment reconnu pour s'imposer dans l'organisation, ils demeurent fortement dépendants du pouvoir exécutif pour s'intégrer dans la collectivité locale.

Cette fragilité statutaire se trouve aggravée par un contexte financier difficile pour l'ensemble des fonctionnels. II se manifeste dans les budgets des collectivités locales dès 1992. Sous l'influence de la décentralisation et des transferts de compétences qui l'accompagnent, le poids des prélèvements obligatoires afférents aux collectivités locales a fortement augmenté. Depuis la décentralisation de 1982, la progression a été

1 Toutefois, des exemples sont antérieurs comme Bordeaux (1966), Nice (1967), Lille, Toulouse, Metz.

2 Langenieux-Villard (P.), L'information municipale, Paris, PUF, coll. Que sais-je ?, 1985, pp. 16-19; Boure (Robert), "Quand les collectivités territoriales entrent en communication", CinémAction, n63, mars 1992, pp.169-174.

3 Poujade (Marie-Yvonne), La formation dans les collectivités locales - Les moyens d'une politique, CNFPT, 1994, p. 83.

4 Crozier (Michel), Etat moderne, Etat modeste, Paris, Fayard, 1987, p. 288, où l'administration d'Etat agit de façon similaire.

5 Poujade (Marie-Yvonne), ibid. 
de $8,5 \%$ l'an, soit trois points de plus que le PIB. Le rythme s'atténue dans les années quatre-vingt-dix avec une progression annuelle de 5 a $6 \% 1$.

Dans cette recherche d'économie de fonctionnement, l'action des fonctionnels récents peut se voir contestée. Ainsi, les opérations de communication peuvent être remises en cause notamment par l'opposition ${ }^{2}$ ou de façon générale 3 . Globalement la progression des budgets de communication et de formation s'est ralentie 4 . Cependant, cette contrainte financière n'a pas eu pour conséquence de remettre en cause la légitimité méme de ces fonctions récentes. Elle a surtout fait sentir ses effets dans la ventilation des dépenses. Ces dernières ont été concentrées sur des actions d'allure moins dispendieuses à la visibilité plus marquée dans le court-terme. Dans le domaine de la formation, les actions d'adaptation aux postes de travail ont pris le pas sur une logique de gestion prévisionnelle des emplois et des métiers.

Dans les deux cas, le pouvoir exécutif a donné la priorité aux développements les plus rapidement tangibles. C'est pourquoi, la capacité à faire émerger leurs contributions au fonctionnement de l'organisation devient un enjeu pour ces fonctions, non pas pour demeurer dans l'organisation (nous avons pu voir que la question ne se pose pas) mais pour renforcer, voire étendre leur influence. Aussi, la question de l'évaluation de leurs actions se pose.

\section{- L'évaluation : une méthodologie introuvable pour une démarche intenable}

Les responsables tant de la formation que de la communication insistent sur le fait qu'ils doivent faire aussi bien avec moins. Le souci de l'évaluation de leurs actions leur apparaît comme plus présent, voire pressant dans leur pratique professionnelle. Ces fonctions doivent rendre perceptible leur contribution pour apparaitre comme un investissement6, c'est-à-dire comme une activité sur laquelle l'élu peut capitaliser et obtenir un retour sous la forme d'une amélioration du fonctionnement de l'organisation et de la perpétuation de son pouvoir politique. Or, la démarche est problématique. Elle se fonde sur des méthodes mal assurées, diverses. Elle est confrontée à la nature complexe du travail de fonctionnels. Par ailleurs, elle conduit à interroger trop directement les prérogatives de l'élu pour être systématisée et aboutir techniquement.

1 Boeuf (Jean-LuC), "Quinze ans de décentralisation", Problèmes politiques et sociaux, n787, 11 juillet 1997, pp. $10-17$.

2 Dubois (Francoise), Les politiques de communication des collectivités territoriales : le cas de la Picardie, these pour le Doctorat de Science Politique, université de Picardie-CURAPP, 1993, $486 \mathrm{p}$.

3 Un dirigeant de l'agence Euro RSCG Public (actuellement Euro RSCG Corporate) interrogé le signale à propos de la communication pour les actions sociales : la critique porte sur les dépenses de communication qui auraient pu servir à aider les populations nécessiteuses.

4 Poujade (Marie-Yvonne), op. cit. : "Dossier communication publique - Budgets : Une mairie sur deux touchée par les restrictions", CB News, n'459 du 2 au 8 décembre 1996, pp. 54-56.

5 Gerbet, "Le responsable de formation : faire mieux avec moins", Personnel, 1994.

6 Caspar (Pierre), Meiganant (Alain), "L'investissement formation un mythe utile", Education permanente, n'9, oct 88, pp. 11-20. 
En effet, les fonctions transversales se heurtent à la très grande difficulté consubstantielle de l'évaluation de leurs effets pris isolément. "I s'agit toujours d'actions de service liées à d'autres directions administratives. C'est l'idée qu'exprime Gadrey lorsqu'il définit le service comme "... visant une transformation d'état d'une réalité $C$, possédée ou utilisée par un consommateur (ou client, ou usager) $B$, réalisée par un prestataire $A$, à la demande de $B$, et souvent en relation avec lui, mais n'aboutissant pas à la production d'un bien susceptible de circuler économiquement indépendamment du support $\mathrm{C}$ " . Au total, ces activités de service s'insèrent dans une logique de co-production, voire de co-responsabilité2 rendant difficile l'identification de la contribution des fonctionnels.

Il en découle que la grille classique d'évaluation gestionnaire fondée sur le principe de maîtrise et d'isolement de la contribution, s'avère inopérante à saisir l'apport réel des services transversaux ${ }^{3}$. On se heurte à une absence de consensus sur les méthodes à employer, en raison de la dimension récente de ces activités et de leur transversalité. Le décalage entre le discours ambitieux de l'évaluation, répondant à la volonté de s'insérer dans le cadre financier dominant et les pratiques est important. Dans le champ de la formation, depuis les travaux de Kirkpatrick4, de nombreuses méthodes ont été proposées et mises en place dans différentes organisations. Or, les résultats demeurent modestes 5 . Les indicateurs de productivité utilisés ne font pas ressortir l'accroissement de compétences engendré par une action de formation6. La communication se situe dans une configuration similaire, alors même que les propositions de méthodes d'évaluation sont très variées, voire hétéroclites7. II apparaît qu'il n'y a pas de savoirs stabilisés en la matières.

Plus globalement, on note une résistance que l'on peut qualifier de culturelle au sein des administrations à l'encontre des opérations d'évaluation. Celles-ci ne représentent pas une préoccupation beaucoup plus développée dans l'ensemble des politiques publiques, au point d'être quasiment absentes au sein de l'Etat central en France jusqu'à récemment. L'existence des grands corps de l'Etat a contribué à faire considérer comme superflus des contrôles concurrents 9 . Ces derniers restent

1 Gadrey (J.), L'économie des services, La Découverte, Paris, 1992.

2 Malleret-Dumontant (Véronique), op. cit.

3 Lorino (P.), L'économiste et le manager, Editions de la Découverte, 1991 ; Delaunay (Jean-Claude), "Services complexes : comment optimiser leur rentabilité pour l'entreprise", Revue Française de Gestion, pp. 48-54.

4 Kirkpatrick (D. L.), "Technics for evaluating training programs", Journal of ASTD, vol 13, n¹1-12, 1965.

5 Vial (Philippe), "Les enjeux de l'évaluation de la formation", Actualité de la Formation Permanente, $n^{\circ} 140, p p$. 23-28.

6 Tannenbaum (S-I), Woods (S-B), Determining a strategy for evaluating training : operating within organisational constraints". Human Ressources Planning, vol 15, n², 1992.

7 Pour plus de détails sur les raisons des faiblesses de l'évaluation, v. Bessières (Dominique), op. cit., pp. 430446.

8 Duran (Patrice), Monnier (Eric), "Le développement de l'évaluation en France, nécessités techniques et exigences politiques", Revue Française de Science Politique, vol. 42, n², avril 1992, pp. 235-262.

9 Muller (Pierre), Les politiques publiques, Paris, PUF, coll. Que sais-je ?, 1994, p. 121. 
marginaux aujourd'hui encore, comme l'atteste le Comité interministériel de l'évaluation qui a mis en œuvre seulement trois nouvelles évaluations depuis 19941.

Au-delà de ces contingences techniques, il apparaît que la question de l'évaluation de la performance de ces services fonctionnels à la discrétion du pouvoir ne peut être fondamentalement posée au sein des organisations. En effet, le questionnement financier des services fonctionnels remet indirectement en cause l'action de l'élu. Ces services, bien qu'étant positionnés comme des prestataires de services internes, concrétisent une manifestation de l'emprise du pouvoir central sur le fonctionnement de l'organisation. Du point de vue du contrôle de gestion, ces services transversaux s'analysent comme des centres de coûts discrétionnaires?. On se situe dans des structures juridiquement organisées suivant le principe hiérarchique, dans le cadre d'activités bureaucratiques au sens weberien?3. Cela implique un décideur pour partie extérieur à l'organisation ${ }^{4}$, ce qui est le cas des édiles locaux. Dès lors, tenter d'évaluer leurs actions, revient à prononcer un jugement sur le travail de l'élu, on hésite à l'entreprendre.

Ne pouvant se fonder sur une reconnaissance issue de l'antériorité au sein des collectivités locales, confrontée à des exigences de visibilité de leurs actions, l'intégration des services de communication et de formation s'avère difficile. Si elle n'est pas remise en cause pour l'heure, en revanche leur positionnement au sein de l'organisation demeure en débat. II fait l'objet de lectures plus ou moins favorables suivant la succession des équipes exécutives et l'intérêt économique qu'elles peuvent porter à ces thèmes. Les risques de marginalisation par externalisation quasi complète de ces activités ne sont pas absolument écartés. Leur pérennisation en tant que fonctions associées à la dynamique des administrations décentralisées n'est pas totalement assurée. Elle s'inscrit dans la durée et s'appuie sur différents moyens de légitimation.

Les voies d'une légitimation professionnelle

L'insertion et la reconnaissance de ces nouvelles fonctions met en jeu différentes sources de légitimités. Elles tiennent aux dirigeants, à l'action des services dans l'organisation et à des reconnaissances extérieures.

Pour appréhender ce processus de légitimation, il convient de ne pas le réduire à sa manifestation la plus forte dans une bureaucratie administrative : la primauté de la volonté du dirigeant politique. II est nécessaire de l'aborder suivant une logique

1 Leca (Jean.), Thœnig (Jean-Claude), "Evaluer pour gouverner autrement", Le Monde, mardi 9 décembre 1997, p.V.

2 Malleret-Dumontant (Véronique), op. cit.

3 Weber (Max), Economie et société, Tome Premier, Paris, Plon, 1971, pp. 226-227.

4 C'est la direction administrative bureaucratique avec un détenteur du pouvoir issu notamment de l'élection, idem. 
combinatoire où les différents registres s'articulent suivant des modalités propres à chaque organisation. Pour autant, des lignes de forces peuvent être définies au-delà de la diversité intrinsèque des différentes configurations. Néanmoins, l'appui du sommet hiérarchique constitue la clé de voûte de l'ensemble des équilibres possibles.

\section{- Une légitimité fondée sur la proximité du pouvoir}

Les services de communication et de formation sont proches des décisionnaires des collectivités locales. Le service de formation est rattaché de manière directe (secrétariat général ou directeur général) ou indirecte (service du personnel ou DRH) ${ }^{1}$ aux instances de direction des collectivités locales. Ce positionnement est encore plus marqué pour les services de communication rattachés très majoritairement au cabinet ou à l'exécutif. Ceci les différencie de la communication des ministères et des administrations centrales. Ces derniers ont majoritairement opté pour une communication déconcentrée avec la présence de chargés de communication dans les services administratif̧. II en résulte une légitimation de ces fonctions par la voie hiérarchique, principe d'organisation du droit administratiß.

Leurs missions stratégiques sont d'asseoir le contrôle du pouvoir central sur l'ensemble de l'organisation. Les deux fonctions agissent sur des registres distincts. La formation a pour rôle de maintenir une certaine cohérence dans la perception des stratégies souhaitées par la direction à travers une action indirecte. C'est l'un des rares espaces où la hiérarchie supérieure peut diffuser ses messages à l'ensemble du personnel. Les formations de management ou plus globalement à vocations comportementales constituent un outil de mobilisation de l'encadrement. Les possibles déformations liées aux circuits d'information présents dans les différents niveaux hiérarchiques se trouvent écartées. Au surplus, la direction générale bénéfice de l'image de neutralité dont est encore porteuse la fonction formation. La formation s'avère être un vecteur pouvant permettre d'adjoindre aux principes hiérarchiques une dimension d'adhésion.

Dans le même ordre d'idée, l'insertion dans la structure administrative du service de la communication démontre la volonté du sommel hiérarchique de contrôler plus finement l'organisation. Elle exprime une fonction de centralité à l'échelle de l'organisation. La communication institutionnelle extérieure exercée par un tel service sert à donner une vision de cohérence. En effet, cette activité est contrôlée, monopolisée, par un service qui détient les outils, l'expertise. Un tel contrôle est de nature à servir l'unité de l'organisation de la collectivité locale dans une dimension symbolique produite par et pour sa visibilité extérieure. Cette monopolisation permet

1 Poujade (Marie-Yvonne), op. cit, pp. 13-14.

2 En effet, dans tous les services pour $46 \%$, dans la plupart pour $23 \%$, dans quelques uns $16 \%$, contre $15 \%$ dans aucun service, selon les organismes interrogés par le CECCOPOP en 1995, L'inflation des communicateurs politiques et publiques : symphonie ou cacophonie ?, 1995, p. 23, et pp.17-18.

3 Weil (Prosper), Le droit administratif, Paris, PUF, coll. Que sais-je ?, 1991 (14e édition, 1964), 127 p. 
aux élus de ne pas encourir les risques d'une dispersion des communications institutionnelles entre les différents services administratifs. De telles configurations étaient fréquentes dans les années soixante-dix et au début des années quatre-vingt. On peut y voir une manifestation de l'habitus des hommes politiques soumis à une "contrainte de cohérence" de leurs prises de position', impératif pour organiser la cohésion entre les divers traits de leurs rôles?2. Elle participe d'un mode de gestion des collectivités territoriales issu de la décentralisation, dans lequel l'exercice du pouvoir exclut largement la participation des citoyens et s'appuie sur une politique de communication ${ }^{3}$. On voit clairement que la communication représente un marqueur de pouvoir et un outil pour le chef de l'exécutif, afin d'imposer son autorité sur la structure administrative composée de fonctionnaires protégés par leur statut.

Cependant, ce contrôle centralisé ne peut et ne veut être complet. Seules les actions perçues comme les plus importantes par les élus font l'objet d'une centralisation. C'est pourquoi les fonctions jugées subalternes sont largement déléguées. La centralisation conduit à un double mouvement : sur-qualification du centre des fonctions qui s'accompagne d'une déqualification de ses éléments périphériques. La fonction de formation se structure autour d'une unité centrale, seule détentrice d'un savoir-faire organisationnellement affirmé. Cela lui permet de jouer un rôle d'expertise à l'égard des relais déconcentrés. Ceux-ci sont souvent absorbés dans des postes de généralistes de la fonction ressources humaines. Plus rarement, des cadres opérationnels prennent en charge cette dimension à temps partiel. Ils ont la responsabilité de la mise en œuvre concrète des actions de formation. De façon similaire, des fonctions de communication sont perçues comme secondaires en raison des populations particulières qu'elles traitent (comme les fonctionnaires pour la communication interne) ou restreintes (comme les usagers du minitel, en 1990 seulement $20 \%$ des abonnés au téléphone étaient équipés d'un minitel 4 ). Souvent, elles ne sont pas rattachées à la direction de la communication. Lorsqu'elles le sont, elles disposent de peu de personnels et de budgets, ou elles font l'objet d'une soustraitance.

Cette proximité du pouvoir permet aux responsables de la communication et de la formation d'exercer leurs missions au sein de la collectivité locale. Ils agissent sous l'égide de l'application du principe hiérarchique. Néanmoins, afin d'initier une dynamique propre, garantie d'une intégration plus pérenne dans l'organisation, il convient que ce premier moyen de légitimation soit combiné avec des registres de reconnaissances différents. Quelles que soient les articulations établies, la proximité du pouvoir en constituera le pivot incontournable.

1 Gaxie (Daniel), Lehingue (Patrick), Enjeux municipaux, Paris, PUF/CURAPP, 1984, p. 60.

2 Cette cohésion entre le passé politique et le stéréotype d'un élu proche de ses administrés, compétent et efficace, doit être mis en scène, Briquet ( $\mathrm{J} . \mathrm{L}$. ), "Communiquer en actes", in "Le métier d'élu : jeux de rôles", Politix, n²8, déc. 1994, p. 20.

3 Grémion (Catherine), "De la participation à la communication", in "La communication publique", Revue Française d'Administration Publique n'58, avril-juin 1991, p. 272.

4 Castagnet (Michel), "Développement des phénomènes de communication et participation à la vie locale", Paris, Conseil économique et social, JO de la RF, n4159, 14 février 1991, p. 41. 


\section{- Une légitimité fondée sur une logique de service}

Les services fonctionnels mettent en œurre une logique de "client-fournisseur" interne. Ils deviennent des prestataires de service. Différents avantages sont proposés aux senvices administratifs. Ils contribuent à améliorer tant leur fonctionnement interne (la formation continue) que leur crédibilité au sein de l'organisation dans leur environnement pertinent (la communication). Le bénéfice peut être en premier lieu matériel.

Le service de la formation continue, par de nombreuses visites sur le terrain, par une écoute clientèle forte, peut démontrer la qualité de ses prestations à la hiêrarchie intermédiaire. II lui prouve sa capacité à former son personnel selon les besoins qui ont été définis en commun et dans des délais relativement réduits'. Cette logique marketing démontre aux services opérationnels que la formation continue interne, grâce à son professionnalisme et à sa connaissance intime du fonctionnement de l'organisation, peut rivaliser avec un organisme externe. Ceci vaut également pour la communication. Sa direction peut exercer une dimension de prestataire de services en contrepartie de la vérification de la cohérence des communications des directions administratives. Elle leur permet de profiter des marchés d'impression et d'affichage qu'elle a passés pour une certaine durée. Grâce à son budget, la direction de la communication peut faire office d'agence intégrée ou réaliser directement leurs rédactionnels.

Au demeurant, les services de formation continue et de communication agissent comme de puissants réducteurs de coûts pour les services opérationnels qui désirent mener une action de manière plus autonome. Dans le champ de la formation, le service central connaît mieux le marché des intervenants extérieurs. II peut même avoir établi des relations de partenariat avec certains d'entre-eux, cela se traduit par des tarifs préérentiels. Pour la communication, les services centraux peuvent faire profiter les services administratifs de leurs contacts avec des agences de conseil en communication.

Le bénéfice est aussi symbolique. Cette dimension est plus particulièrement manifeste pour les actions de communication. En effet, la politique de communication constitue un référent central de nature à donner cohérence aux différentes facettes des institutions locales et à leurs actions plurielles, souvent partagées entre différents échelons territoriaux. Diffuser des informations tend à accréditer une image d'efficacité. En conséquence, cela légitime l'action administrative et le pouvoir politique. Les services administratifs par ce biais voient certaines de leurs actions acquérir une visibilité par leur présence dans les supports de communication : magazines, affiches, dépliants. De même, lors de changements organisationnels, le service de formation peut permettre aux services administratifs d'accroittre leur visibilité au sein de la structure, par des moyens informels tels que le bouche-à-oreille ou des présentations plus formalisées.

1 Voir étude du CNFPT sur la professionnalisation du poste de responsable de formation, Juin 1998. 
Néanmoins, il convient de rester mesuré sur l'application de cette logique de service au sein des collectivités territoriales. Ce principe de légitimité doit être associé avec le principe hiérarchique. II ne s'agit pas d'un véritable marché. Nous nous trouvons dans une situation de monopole. Les services internes sont tenus de recourir aux experts de la collectivité territoriale pour les domaines de la formation continue et de la communication. Le non respect de cette règle peut faire réapparaître le principe hiérarchique. Ce dernier s'exerce alors par la combinaison de deux modalités principales. La première est constituée de notes de rappel à l'ordre. La pression peut se faire plus intense et prendre une forme financière. Le service incriminé se voit contraint de prendre à sa charge le coùt du prestataire de services externe. Cependant, certaines collectivités locales laissent à leurs services administratifs des marges de manœuvre. Celles-ci sont encadrées. Des seuils financiers d'intervention sont mis en place. Au-delà d'un certain niveau de dépenses, la direction centrale ne prend pas en charge la réalisation de l'action de formation ou de la communication. Néanmoins, une surveillance s'exerce à travers l'utilisation d'un visa par exemple. Toutefois, ces principes internes de légitimation se trouvent renforcés par la mise en œuvre de logiques extérieures de renforcement.

\section{- Les modes de reconnaissance externes}

Ces modes sont pluriels. Ils recouvrent à la fois des enjeux sociétaux ou législatifs, mais également sociaux dans l'optique de la construction d'un groupe professionnel'.

Le cheminement vers l'affirmation de nouvelles fonctions dans l'organisation se fonde sur l'émergence de valeurs sociétales favorables. C'est particulièrement le cas pour la formation professionnelle, qualifiée de "deuxième chance" par le premier ministre Michel Rocard au début des années 1990. En atteste l'augmentation considérable des sommes imparties sur ce secteur décentralisé par les régions et l'Etat qui a fortement réinvesti le domaine. Ainsi, la région gérait $25 \%$ des crédits de la formation professionnelle au début des années quatre-vingt contre $10 \%$ aujourd'hui parce que l'Etat a accru sa participation?.

La communication à la fin des années soixante-dix et au début des années quatrevingt s'est étendue à tous les espaces sociaux, tout en augmentant ses domaines d'intervention dans chacun d'eux. Les exemples du développement de la fonction relations humaines dans les entreprises et l'élargissement du champ du marketing (les produits, l'institutionnel, le politique) ${ }^{3}$ le montrent. Divers éléments théoriques, idéologiques, économiques et sociaux se conjuguent pour faire de la communication une valeur et une croyance de la société. La force de la communication réside dans

1 Chapoulie (Jean-Marie), "Sur l'analyse sociologique des groupes professionnels", Revue Française de Sociologie, XIV, 1973, pp. 86-114.

2 Rémond (René), "René Rémond : 'la décentralisation, qui permet de vivifier notre système de représentation constitue une réponse à la crise actuelle..." (propos recueillis par J-M. Ohnet), Pouvoirs Locaux, n³, 4 ème trimestre 1989, p. 91.

3 Sfez (Lucien), Critique de la communication, Paris, Seuil, coll. Empreintes, 1988, pp. 21-22, p. 26, p. 279. 
ses analyses théoriques, ses exemples de pratiques. Elle tend à être présentée comme un impératif. Sa diffusion dans tous les champs, selon des rythmes spécifiques, fait que la société est conquise dans toutes ses dimensions par cette pratique et ce thème ${ }^{1}$. Au total, la société de communication réfère à "une signification imaginaire centrale" de nature mythique. Ce mythe ${ }^{2}$ prend racine dans les techniques de communication objectives et parvient à regrouper "...des redéfinitions des savoirsfaire et des pratiques dans les espaces sociaux les plus variés" 3 . Ces préoccupations sociétales sont partiellement traduites en termes légaux.

La reconnaissance s'exprime aussi par le biais du droit. Le législateur possède le pouvoir de conditionner la réalité. Issue du mouvement d'éducation populaire porté par l'action de Léo Lagrange lors du Front populaire, la formation professionnelle continue intègre le monde des organisations par le biais de la loi du 16 juillet 1971. Elle vise trois objectifs principaux : favoriser les possibilités d'adaptation des employés au cours de leur vie professionnelle, lutter contre les inégalités des chances, reconnaître à l'entreprise une capacité de formationt. La loi reconnaît une pratique existante en créant des obligations à l'égard des organisations et en légitimant ces préoccupations à la fois pour le secteur privé et le secteur public (un pour-cent de cotisation au $\mathrm{CNFPT}^{5}$ de la masse salariale doit y être consacré) 6 . Dans le même ordre d'idée, les possibilités de communication des collectivités locales avant les élections sont limitées en vertu de la loi du 15 janvier 1990. La communication locale ne doit plus être personnalisée au moins six mois avant le scrutin, ni susceptible d'une campagne de promotion publicitaire des réalisations ou de la gestion?. Les restrictions apportées par la loi sont de nature à renforcer la légitimité de la communication locale en contribuant à éliminer la communication au profit direct des élus pour une communication d'apparence publique.

Ces prescriptions légales sont intégrées dans la pratique des organisations. Le management public dirigé vers l'action "ne peut ignorer le droit quand celui-ci se définit comme une discipline d'action à visée normative" 8 . Max Weber a souligné "ce qui détermine la 'validité' d'une prescription, ce n'est pas le fait qu'elle soit 'observée', mais le fait que certaines activités soient 'orientées' en fonction d'elle" ${ }^{m}$, particulièrement en

1 Miege (Bernard), La société conquise par la communication, Grenoble, PUG, 1989, pp. 143-164.

2 Nous reprenons la double dimension donné par Erik Neveu à la notion de mythe qui insiste sur sa dimension magique qui enracine une croyance et impose un sens rassurant reliant des phénomènes sociaux épars, Une société de communication ?, Paris, Montchrétien, coll. Clefs politique, 1994, pp. 70-71 et p. 87.

3 Neveu (Erik), "La société de communication et ses interprètes", Réseaux, n64, mars-avril 1994, CNET, Paris, p. 11.

4 Delors (Jacques), "Genèse d'une loi et stratégie du changement", Connexion, n०17, 1976, pp. 39-50; Meignant (Alain) et Palazzeschi (Yves), "La loi du 16 juillet 1971 en gestation", Personnel, $n^{\circ} 378$, mars avril' 1997.

5 Centre National de la Fonction Publique Territoriale.

6 Poujade (Marie-Yvonne), op. cit.

7 Bessières (Dominique), op. cit, pp. 409-414.

8 Duran (P.), "Piloter l'action publique, avec ou sans le droit", Politiques et Management Public, vol. 11, $n^{\circ} 4$, décembre 1992, p. 6.

9 Weber (Max), Economie et société, Tome Premier, Paris, Plon, 1971, p. 323. 
matière d'action publique dominée par le droit 1 . Leurs applications concrètes relèvent de l'expertise des professionnels de la communication, dont la présence est rendue plus nécessaire en raison des risques, notamment de remise en cause des élections en cas de manquements. Plus largement, l'activité de ces deux fonctions est régie par une norme impersonnelle qui les légitime en définitive 2 . Les constitutions de collectifs se voulant "représentatifs" de ces professionnels œuvrent au même processus.

Avec les associations, la logique de renforcement du groupe est clairement perçue. Elles ont pour trait commun de se présenter et de se positionner comme une lieu d'échanges entre professionnels, d'apprentissage selon les dires des deux dirigeants des associations "Communication et Citoyens" et "Communication Publique"3 pour la communication, le "Groupement des Animateurs et Responsables de Formation" et I'Association Française des Responsables de Formation" pour la formation. Elles réalisent des études et préconisent un certain nombre d'actions en matière de communication locale et de formation. Elles œuvrent à une réflexion déontologique. L'amélioration instrumentale est visée. Elles se doublent d'une recherche de renforcement de l'intégration dans la structure administrative par une reconnaissance interne, pendant d'une reconnaissance et d'une légitimation externe de nature associative. Elles ont vocation à produire ainsi un discours sur la profession, à solidifier le champ professionnel. II s'agit de viser une amélioration de l'instrument de la communication et de la formation, afin de conforter la légitimité des professionnels praticiens vis-à-vis des élus.

Ainsi, l'association "Communication Publique" exerce un travail de fond. Sa visibilité d'association relève en grande partie des publications et des ouvrages publiés au nom ou sous l'égide du président fondateur de l'association, Pierre Zémor4, Conseiller d'État, ce qui ajoute à la légitimation de l'activité par le patronage d'un membre d'un grand corps de l'Etat. Du fait même de son existence et de ses activités, l'association participe donc à l'affirmation et à la recherche des spécificités d'une communication publique et, au sein de celle-ci, de la communication publique territoriale5. De façon plus globale, dans les associations professionnelles, un discours est produit et répété dans les différents écrits et dans les différentes réunions, rencontres, colloques. Des valeurs sont ainsi affichées, elles sont destinées à servir de références identitaires, au

1 Chevallier (Jacques), "La juridicisation des préceptes managériaux", Politiques et Management Public, vol 11. $n^{\circ} 4$, décombre 1993. p. 112.

2 Laufer (Romain.), Paradeise (Catherine), Le prince bureaucrate, Paris, Flammarion, 1982, pp. $42-43$.

3 La première étant exclusivement consacrée aux acteurs de la communication locale, membres de collectivités locales ou d'agences. La seconde exclusivement ouverte aux communicants publics et fermée aux agences.

4 L'association est à l'origine de différentes publications telles que "La communication publique", Revue française d'administration publique (1991), La communication publique en pratique (sous le pseudonyme de Marianne Messager - 1994). Ces deux publications sont préfacées ou introduites par Pierre Zémor. Celui-ci est l'auteur de nombreux articles dans la presse ou dans des revues, d'un rapport au Ministre Jean-Pierre Soisson sur l'organisation et le fonctionnement de la communication des institutions de service public intitulé Le sens de la relation (1992), ainsi que d'un "Que sais-je ?" intitulé La communication publique (1995).

5 Un colloque sur la communication des départements est particulièrement illustratif des opérations de légitimation que de telles associations peuvent construire, v. Bessières (Dominique), Ollivier-Yaniv (Caroline), "La communication départementale : constat d'une Arlésienne ou processus de légitimation ?", Quaderni, n³1, hiver 1997, pp. 19-31. 
moins pour les membres du réseau. Ce faisant, elles œuvrent à l'élargissement de leur champ de compétences, et donc à l'affirmation de leur influence.

Cercles d'échanges, de confrontations d'expériences, d'études et d'actions de lobbying, les associations professionnelles constituent un moyen privilégié d'accession à la reconnaissance sociale pour un métier. Comme le rappelle Chapoulie, elles constituent "une garantie de l'existence et de l'unité dans la réalité sociale des professions, dans la mesure où elles sont à l'origine, sinon de la production, du moins de la systématisation et de la diffusion du stéréotype de la profession ${ }^{\natural}$. Les métiers de responsables de la communication et de la formation ne font pas exception à cette règle. Dans les deux cas, un travail d'institutionnalisation, de construction d'un discours identitaire est mené par ces groupements professionnels auprès des pouvoirs publics.

\section{Conclusion}

Les mécanismes de légitimation des activités fonctionnelles transversales récentes sont variés. Ils sont d'autant plus importants que leur intégration est contestée et leurs résultats difficilement évaluables. Dans le cadre des collectivités locales, nous avons discerné trois grands registres de légitimation. Le premier, le plus spécifique et le plus fort, est une légitimité issue du pouvoir de l'élu. Il est le décideur final de l'implantation de ces fonctions ayant vocation à exercer un contrôle organisationnel latent. Le second, le plus nécessaire à la reconnaissance pratique dans les organisations, repose sur une logique "client-fournisseur". Celle-ci valorise l'utilité organisationnelle de ses activités auprès des directions administratives. Enfin, l'environnement extérieur sociétal, juridique ou professionnel, peut être un support efficace de légitimation. En intégrant dans l'organisation des préoccupations issues de la société, il oriente son fonctionnement interne au bénéfice de la formation et de la communication.

Cette étude n'est pas exhaustive. Elle permet néanmoins de proposer des pistes de réflexion sur les conditions d'émergence, d'insertion et de pérennisation des fonctions transversales se présentant comme coopératives. En effet, tant la formation que la communication sont perçues au niveau sociétal comme des facteurs devant contribuer à l'ouverture des organisations. Elles apparaissent, au niveau des discours, comme les outils de nouvelles formes de travail plus coopératives, laissant place à un dialogue entre les subordonnés et leur supérieur hiérarchique. Or, nos observations nous conduisent à relativiser fortement cette approche en terme d'"organisation apprenante"2. Loin de répondre à ce cliché, la communication comme la formation ne remettent nullement en cause le modèle hiérarchique. Bien au contraire, elles contribuent à en accroitre la portée comme l'efficacité. Elles mettent sous contrôle des aspects jusqu'ici non intégrés dans le modèle et en limitent les effets internes trop

1 Chapoulie (Jean-Marie), art. cité.

2 Argyris (Christopher), Savoir pour agir. Comment surmonter les obstacles à l'apprentissage organisationnel Interédition, 1995 ; Baumard (Philippe), "Les organisations apprenantes ? Les dangers de la consensualité", Revue Française de Gestion, sept-oct 1995. 
coercitifs. Ainsi, l'autorité se fait moins directe en apparence. En profondeur, dans le fonctionnement de l'organisation, elle se trouve renforcée par ses fonctions transversales. Tout ce passe comme si l'accroissement du pouvoir du sommet hiérarchique était rendu plus supportable par la reconnaissance acquise de ces fonctions. De sorte qu'il s'agit d'un pouvoir reconnu et méconnu en tant que tel. 